WIS-98/24/Sep-DPP

SLAC-PUB-7950

hep-ph/9809524

\title{
Can lepton flavor violating interactions explain the LSND results?
}

\author{
Sven Bergmann ${ }^{a}$ and Yuval Grossman ${ }^{b}$ \\ ${ }^{a}$ Department of Particle Physics \\ Weizmann Institute of Science, Rehovot 76100, Israel \\ ${ }^{b}$ Stanford Linear Accelerator Center \\ Stanford University, Stanford, CA 94309
}

\begin{abstract}
If the atmospheric and the solar neutrino problem are both explained by neutrino oscillations, and if there are only three light neutrinos, then all masssquared differences between the neutrinos are known. In such a case, existing terrestrial neutrino oscillation experiments cannot be significantly affected by neutrino oscillations, but, in principle there could be an anomaly in the neutrino flux due to new neutrino interactions. We discuss how a non-standard muon decay $\mu^{+} \rightarrow e^{+} \bar{\nu}_{e} \nu_{\ell}$ would modify the neutrino production processes of these experiments. Since $S U(2)_{L}$ violation is small for New Physics above the weak scale one can use related flavor-violating charged lepton processes to constrain these decays in a model independent way. We show that the upper bounds on $\mu \rightarrow 3 e$, muonium-antimuonium conversion and $\tau \rightarrow \mu e e$ rule out any observable effect for the present experiments due to $\mu^{+} \rightarrow e^{+} \bar{\nu}_{e} \nu_{\ell}$ for $\ell=e, \mu, \tau$, respectively. Applying similar arguments to flavor-changing semi-leptonic reactions we exclude the possibility that the "oscillation signals" observed at LSND are due to flavor-changing interactions that conserve total lepton number.
\end{abstract}

Research at SLAC is supported by the U.S. Department of Energy under contract DE-AC03-76SF00515 


\section{INTRODUCTION}

There are strong experimental hints that suggest that the neutrino sector is more complicated than it is in the Standard Model. In particular, the atmospheric neutrino anomaly [i] and the solar neutrino problem [商] can be explained with massive neutrinos.

The atmospheric neutrino anomaly [i] i i the observation that the ratio of muon neutrinos to electron neutrinos that are produced in the atmosphere is about 0.6 of the theoretical expectation assuming Standard Model neutrinos. Recently, the Super-Kamiokande Collaboration has published [3i3] the analysis of their atmospheric neutrino data from a 33.0 kiloton-year (535-day) exposure. The data exhibit a zenith angle dependent deficit of muon neutrinos which cannot be explained with the Standard Model massless neutrinos. The estimated probability that the observed $\mu / e$ ratio could be due to statistical fluctuations is less than $10^{-5}$ (for the sub-GeV data), which is widely considered as the first "proof" for

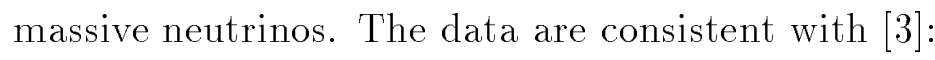

$$
5 \times 10^{-4} \mathrm{eV}^{2}<\Delta m^{2}<6 \times 10^{-3} \mathrm{eV}^{2} \quad \sin ^{2} 2 \theta>0.82 \quad(90 \% \text { C.L. }),
$$

where $\Delta m^{2}$ is the mass-squared difference and $\theta$ is vacuum mixing angle for the favored $\nu_{\mu} \leftrightarrow \nu_{\tau}$ oscillations. Note that $\nu_{\mu} \leftrightarrow \nu_{e}$ oscillations are disfavored by the observed zenith angle distribution and by the fact that the up-to-down ratio for $\nu_{\mu}$-induced events departs much more from unity than for the $\nu_{e}$-induced events. Moreover the CHOOZ experiment [Ait] independently rules out $\bar{\nu}_{e} \leftrightarrow \bar{\nu}_{\mu}$ oscillation for mixing as large as in (1. $10^{-3} \mathrm{eV}^{2}$.

The long standing solar neutrino puzzle $\left[\begin{array}{l}\overline{2} \\ \underline{1}\end{array}\right]$ is now confirmed by five experiments using three different experimental techniques and thus probing different neutrino energy ranges. All these experiments observe a solar neutrino flux that is smaller than expected. The most plausible solution is that the neutrinos are massive and there is mixing in the lepton sector. Then neutrino oscillations can explain the deficit of observed neutrinos with respect to the Standard Solar Model. In the case of matter-enhanced neutrino oscillations, the famous MSW effect provides an elegant solution to the solar neutrino problem. The best fit is obtained for the small angle solution which is given by [2]

$$
\Delta m^{2}=5.4 \times 10^{-6} \mathrm{eV}^{2}, \quad \sin ^{2} 2 \theta=6.0 \times 10^{-3},
$$

where $\theta$ is vacuum mixing angle in a two active neutrino framework involving the $\nu_{e}$ and either $\nu_{\mu}$ or $\nu_{\tau}$. The large angle solution can also explain the data (with a worse fit) with $\Delta m^{2}=1.8 \times 10^{-5} \mathrm{eV}^{2}$ and $\sin ^{2} 2 \theta=0.76$. Finally, vacuum oscillations provide an alternative solution with the best-fit solution [는] given by $\Delta m^{2}=8.0 \times 10^{-11} \mathrm{eV}^{2}$ and $\sin ^{2} 2 \theta=0.75$.

It is well known that the Standard Model contains only three generations of neutrinos and that SLD and LEP data exclude the existence of a fourth light sequential neutrino [5]. If, indeed, there are only three light neutrinos, then an important consequence of the above 


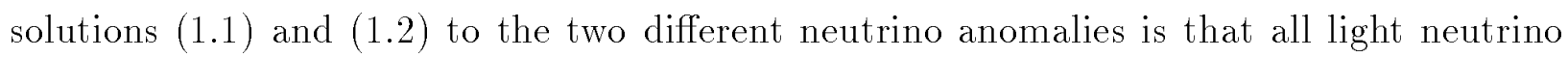
mass-squared differences $\Delta m_{i j}^{2} \equiv m_{i}^{2}-m_{j}^{2}$ are completely determined. The reason is that with three generations, there are only two independent mass differences since $\Delta m_{21}^{2}+\Delta m_{32}^{2}=$ $\Delta m_{31}^{2}$. In particular, we learn that for any $i, j=1,2,3$,

$$
\Delta m_{i j}^{2} \lesssim 10^{-2} \mathrm{eV}^{2}
$$

This is below the sensitivity of all existing terrestrial experiments (except the above men-

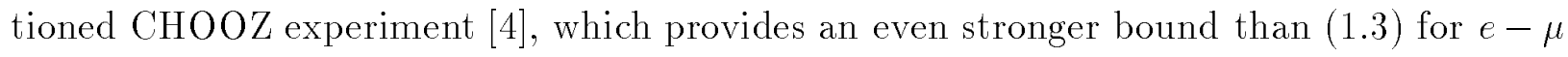
oscillations and large mixing). The conclusion is that if both the atmospheric neutrino anomaly and solar neutrino problem are explained by neutrino oscillations and there are only three light neutrinos, then an extended three generation Standard Model, which allows for small neutrino masses but leaves all interactions as they are in the Standard Model, predicts that no anomaly should be observed in any terrestrial neutrino experiment.

In contrast to this expectation, the LSND collaboration has reported a positive signal in two different appearance channels. The first analysis [i $\overline{6}_{1}^{]}$uses $\bar{\nu}_{\mu}$ 's from muon decay at rest (DAR) and searches for $\bar{\nu}_{e}$ 's via inverse beta decay. The observed excess of $\bar{\nu}_{e}$ events

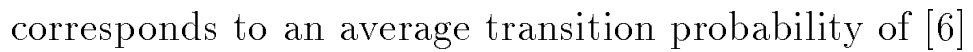

$$
P\left(\bar{\nu}_{\mu} \rightarrow \bar{\nu}_{e}\right)=\left(3.1_{-1.0}^{+1.1} \pm 0.5\right) \times 10^{-3} .
$$

This result by itself could be explained by neutrino oscillations, with $\Delta m^{2}$ and $\sin ^{2} 2 \theta$ in the range indicated in Fig. 3 of Ref. [6-ib]. Taking into account the restrictions from the null results of other experiments, the preferred values of the neutrino parameters are $\Delta m^{2} \approx 2 \mathrm{eV}^{2}$ and $\sin ^{2} 2 \theta \approx 2 \times 10^{-3}$ and the lower limit on $\Delta m^{2}$ for the neutrino oscillation solution is given by

$$
\Delta m^{2}>0.3 \mathrm{eV}^{2}
$$

The second analysis [i] uses $\nu_{\mu}$ 's from pion decay in flight (DIF) and searches for $\nu_{e}$ 's via the $\nu_{e} C \rightarrow e^{-} X$ inclusive reaction. Again, a positive signal has been reported, which could be explained with neutrino oscillations that require neutrino parameters similar to those of the DAR result. However, the statistical significance of this result is much smaller than the one of the DAR analysis.

Obviously, the lower bound (1.5ith solutions to the atmospheric neutrino anomaly (1) (1) and the solar neutrino problem (1) (1) three generation framework. One possibility is to postulate a light "sterile neutrino" [8; Standard Model singlet that mixes with the active neutrinos. Then there would be four neutrino masses which give three independent mass differences, as required to explain the three mentioned results [i] $\left.\overline{0}_{1}^{i}\right]$. Although adding ad hoc this sterile neutrino would be phenomenologically satisfactory, it is not well-motivated to have a light SM singlet. (For attempts to naturally get a light sterile neutrino see e.g., [ii] .) 
Due to the unappealing theoretical feature of a light sterile neutrino, it is interesting to look for alternatives that could explain the LSND anomaly with the known three light neutrinos only. The authors of Ref. [י[1] $\left.\overline{1}_{1}^{1}\right]$ have suggested that the atmospheric neutrino anomaly and the LSND result are explained by the same mass-squared difference. In Ref. [i: 2 a scenario where both the solar neutrino and the atmospheric neutrino anomalies are solved by the same $\Delta m^{2}$ has been studied. While these explanations were marginally consistent at the time, they are excluded by the latest data. (There has been another more recent attempt [1] $\left[\begin{array}{l}1 \\ 2\end{array}\right]$ to explain all experimental data except the Homestake measurement with three active neutrinos only. However their results have been criticized by the authors of [1] $\left.{ }_{1}^{1} \mathbf{u}_{1}^{1}\right]$.)

The aim of this work is to investigate another approach. We assume that the three light neutrinos are not only massive but also interact through lepton flavor violating (but total lepton number conserving) interactions, which are forbidden in the Standard Model. This is an attractive possibility, because various extensions of the Standard Model which predict neutrino masses also give rise to such new interactions. These interactions can affect the LSND production or detection processes or both. We analyze the consequences of small lepton flavor violating interactions for short-baseline neutrino oscillations experiments like LSND under the assumption that all neutrino parameters are fixed to solve the atmospheric neutrino and the solar neutrino anomaly. We find that such a scenario, where new interactions explain the LSND result(s), can be ruled out in a model independent way.

We note that the implications of exotic muon decays on the LSND neutrino production have been studied by Herczeg [1] showing within two explicit models, the left-right symmetric model (LRSM) and SUSY without $R$-parity, that new interactions are too small to be relevant for LSND. More recently the authors of Ref. [i] $\left.{ }_{1}^{1} \overline{\hat{\sigma}_{i}}\right]$ have argued in favor of such a solution (claiming that the DAR result could be explained within LRSMs). However, they seem to have overlooked the strongest experimental bound coming from muonium-antimuonium conversion.

Our paper is organized as follows. In Section 'III'we introduce the formalism to describe the flavor violating interactions. In Section $\left[\begin{array}{l}\mathrm{I} \\ \mathbf{I}\end{array}\right.$ related lepton flavor violating interactions containing only charged leptons. In Section, $\mathbf{I} \mathbf{V}_{!}^{\prime}$ we show how these bounds can be used to derive constraints on $\mu^{+} \rightarrow e^{+} \bar{\nu}_{e} \nu_{\ell}$ within specific extensions of the Standard Model. We generalize this idea in Section 'Vítand show in a model independent way that the anomalous muon decay cannot have a detectable effect in existing terrestrial neutrino oscillation experiments. In Section leptonic reactions and argue that also in this case the bounds on $S U(2)_{L}$ related processes involving only charged fermions can be used to rule out model independently the possibility that lepton flavor violating interaction which conserve total lepton number provide a valid explanation for the LSND results. We conclude in Section :VTI. 


\section{NEUTRINO OSCILLATIONS AND NEW INTERACTIONS}

We start by reviewing the formalism of oscillation experiments in the presence of nonstandard neutrino interactions [1] $\left[\begin{array}{l}1 \\ \mathbf{1}\end{array}\right]$. To illustrate this "hybrid" situation of having both non-trivial neutrino properties and new interactions, we assume two neutrino flavors, CP conservation, that the new interactions have the same Dirac structure as the standard one and that the neutrinos are highly relativistic. In general, in the presence of New Physics, the neutrinos that are produced and detected are not the weak eigenstates. Therefore, we denote these neutrino states by the super-indices $p$ and $d$ which stand for production and detection, respectively. Consider the LSND setting: Antineutrinos are produced by $\mu^{+} \rightarrow e^{+} \nu_{e}^{p} \bar{\nu}_{\mu}^{p}$, and detected by $\bar{\nu}_{e}^{d}+p \rightarrow e^{+}+n$. We define the relevant mixing angles

$$
\sin \theta_{p d} \equiv\left\langle\bar{\nu}_{\mu}^{p} \mid \bar{\nu}_{e}^{d}\right\rangle, \quad \sin \theta_{m d} \equiv\left\langle\bar{\nu}_{2} \mid \bar{\nu}_{e}^{d}\right\rangle, \quad \sin \theta_{m p} \equiv\left\langle\bar{\nu}_{1} \mid \bar{\nu}_{\mu}^{p}\right\rangle,
$$

where $\nu_{1}$ and $\nu_{2}$ are mass eigenstates. Then in the presence of lepton flavor violating interactions, the probability of finding a positron signal in the beam at distance $L$ is [i]

$$
P_{e \mu}^{N}(x)=\sin ^{2} \theta_{p d}+\sin 2 \theta_{m d} \sin 2 \theta_{m p} \sin ^{2} x .
$$

Here $x \equiv \Delta m^{2} L / 4 E$ and we used $E_{1}-E_{2} \approx\left(m_{1}^{2}-m_{2}^{2}\right) / 2 E$, where $E$ is the average energy. In the limit of the Standard Model with massive neutrinos $\left(\theta_{p d}=0\right.$ and $\left.\theta_{m p}=\theta_{m d} \equiv \theta\right)$ eq. (5.2.2.

$$
P_{e \mu}(x)=\sin ^{2} x \sin ^{2} 2 \theta
$$

However, the upper bound (1.3. implies that $\sin ^{2} x \leq \mathcal{O}\left(10^{-4}\right)$ for LSND. Therefore the oscillation part in (12.21) is only a negligible contribution to the required transition probability (1.4) leading to

$$
P_{e \mu}^{L S N D}=\sin ^{2} \theta_{p d}
$$

We learn that the only significant source for the signal seen at LSND is a non-vanishing $\theta_{p d} \neq 0$, namely, the produced (anti)neutrinos are not orthogonal to those that are searched for. We note that from experiments we know that neutrino interactions are dominantly those of the Standard Model. Therefore, while $\theta_{m p}$ and $\theta_{m d}$ may be large, $\theta_{p d}$ has to be small implying that the above appearance probability (2.2.4. must be small.

We first consider new physics effects in purely leptonic interactions. (New physics effects in semi-leptonic processes are studied in section (VIi). Such effects are only relevant for the DAR, where they modify the muon decay. The detection process is given by the Standard Model and therefore is sensitive only to left-handed neutrinos. In that case, the effective interaction for the muon decay is given by $\left[\begin{array}{ll}1 \\ 1\end{array}\right]$ 


$$
\mathcal{H}^{\nu}=\frac{4 G_{F}}{\sqrt{2}}\left[\left(g_{L L}^{V}\right)^{\alpha \beta}\left(\overline{e_{L}} \gamma^{\mu} \nu_{\alpha L}\right)\left(\overline{\nu_{\beta L}} \gamma_{\mu} \mu_{L}\right)+\left(g_{R R}^{S}\right)^{\alpha \beta}\left(\overline{e_{R}} \nu_{\alpha L}\right)\left(\overline{\nu_{\beta L}} \mu_{R}\right)\right]
$$

where the sum over the weak flavor indices $\alpha, \beta=e, \mu, \tau$ is implicit. In the Standard Model the only non-vanishing coefficient is $\left(g_{L L}^{V}\right)_{e \mu}=1$ leading to the standard muon decay

$$
\mu^{+} \rightarrow e^{+} \nu_{e} \bar{\nu}_{\mu}
$$

We define $G_{N}^{\nu}$ to be the effective coupling of the anomalous muon decays

$$
\mu^{+} \rightarrow e^{+} \bar{\nu}_{e} \nu_{\ell}
$$

for $\ell=e, \mu, \tau$ respectively. In terms of the couplings in eq. (2.5.5.

$$
\left|\frac{G_{N}^{\nu_{\ell}}}{G_{F}}\right|^{2}=\left|\left(g_{L L}^{V}\right)_{\ell e}\right|^{2}+\frac{1}{4}\left|\left(g_{R R}^{S}\right)_{\ell e}\right|^{2} .
$$

The three processes in $(2 . \overline{1})$ cannot interfere with each other because they have different final states. Hence the combined effective coupling for muon decays that produce $\bar{\nu}_{e}$ is

$$
\left|G_{N}^{\nu}\right|^{2}=\sum_{\ell}\left|G_{N}^{\nu_{\ell}}\right|^{2}
$$

In terms of $G_{N}^{\nu}$ and for $x \rightarrow 0$ the appearance probability becomes $\left[\begin{array}{l}1 \\ 1 \\ 1\end{array}\right]$

$$
P_{e \mu}=\left|\frac{G_{N}^{\nu}}{G_{F}}\right|^{2} .
$$

From eqs. (1) and (12.1) New Physics coupling should satisfy

$$
r \equiv\left|\frac{G_{N}^{\nu}}{G_{F}}\right|^{2}=\left(3.1_{-1.0}^{+1.1} \pm 0.5\right) \times 10^{-3}
$$

Thus, at the $90 \%$ C.L. we need

$$
r>1.6 \times 10^{-3}, \quad G_{N}>4.0 \times 10^{-2} G_{F} .
$$

In the next section we study the experimental bounds on $r$.

\section{EXPERIMENTAL CONSTRAINTS}

The anomalous muon decay (2. $\left.\bar{z}^{\prime}\right)$ is tightly connected to other lepton flavor violating processes. The Standard Model neutrinos form $S U(2)_{L}$ doublets together with the charged left-handed leptons. As we will show in Section $1 \bar{V}_{1}^{\prime}$ any theory which gives rise to the fourFermi operators that induce the anomalous muon decay $\left(\begin{array}{l}12.5 \\ -2\end{array}\right)$ also necessarily produces the $S U(2)_{L}$ related operators of the form 


$$
\mathcal{H}^{\ell}=\frac{4 G_{V}^{\ell}}{\sqrt{2}}\left(\overline{e_{L}} \gamma^{\mu} \ell_{L}\right)\left(\overline{e_{L}} \gamma_{\mu} \mu_{L}\right)+\frac{8 G_{S}^{\ell}}{\sqrt{2}}\left(\overline{e_{R}} \ell_{L}\right)\left(\overline{e_{L}} \mu_{R}\right)
$$

Here $G_{V}^{\ell}\left(G_{S}^{\ell}\right)$ are the effective New Physics vector (scalar) four-Fermi couplings. Furthermore we define the combined coupling

$$
\left|G_{N}^{\ell}\right|^{2} \equiv\left|G_{V}^{\ell}\right|^{2}+\left|G_{S}^{\ell}\right|^{2}
$$

for $\ell=e, \mu, \tau$, respectively. In general, there might be other interaction terms where all the charged fermions are right-handed. Clearly, such interactions do not relate to those involving neutrinos. We therefore ignore such terms and assume that there is no fine-tuned cancellation between these terms and those we are considering.

The operators in ( leptons. As we shall see the effective couplings $G_{N}^{\nu_{\ell}}$ and $G_{N}^{\ell}$ are always correlated. There is no experimental evidence for any non-vanishing $G_{N}^{\ell}$, so the upper bounds on $G_{N}^{\ell}$ can be used to derive constraints on $G_{N}^{\nu_{\ell}}$. Specifically, the most stringent upper bounds on $G_{N}^{\ell}$ come from $\mu \rightarrow 3 e$ (for $\ell=e$ ), muonium-antimuonium conversion (for $\ell=\mu$ ) and $\tau \rightarrow \mu e e$ (for $\ell=\tau)$. Before we turn to a discussion of the exact relation between $G_{N}^{\nu_{\ell}}$ and $G_{N}^{\ell}$ we present the current experimental bounds on these three lepton flavor violating processes and their implication on $G_{N}^{\ell}$.

Using the upper bound $\operatorname{BR}(\mu \rightarrow 3 e)<1.0 \times 10^{-12}$ together with $\operatorname{BR}\left(\mu \rightarrow e \bar{\nu}_{e} \nu_{\mu}\right)=1$ $[5]$ we obtain

$$
G_{N}^{e} \equiv G_{\mu \rightarrow 3 e}<1.0 \times 10^{-6} G_{F}
$$

The current bound on the muonium-antimuonium conversion effective interaction is [i] $\overline{1} \overline{9}]$

$$
G_{N}^{\mu} \equiv G_{M \bar{M}}<3.0 \times 10^{-3} G_{F}
$$

The upper bound $\operatorname{BR}\left(\tau^{-} \rightarrow \mu^{+} e^{-} e^{-}\right)<1.5 \times 10^{-6}$ together with $\operatorname{BR}\left(\tau^{-} \rightarrow \mu^{-} \bar{\nu}_{\mu} \nu_{\tau}\right)=$ $0.174[$ [n] implies that

$$
G_{N}^{\tau} \equiv G_{\tau \rightarrow \mu e e}<2.9 \times 10^{-3} G_{F}
$$

Note that for New Physics interactions that have a different Dirac structure than those in the Standard Model there exist additional constraints on $G_{S}^{\ell}$ which come from the bounds on the Michel parameters [5. We find that at $90 \%$ C.L.

$$
G_{S}^{\ell}<3.3 \times 10^{-2} G_{F}
$$

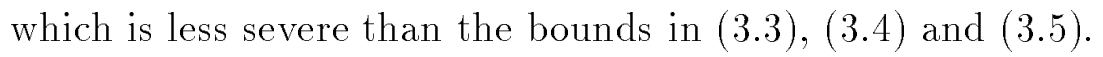

If $S U(2)_{L}$ breaking effects are negligible then $G_{N}^{\ell}$ equals to $G_{N}^{\nu_{\ell}}$ up to a factor of at most two from a possible Clebsch-Gordon coefficient. If we assume moreover that either $G_{N}^{\mu}$ or $G_{N}^{\tau}$ are close to their experimental limit (we will show later that relaxing these assumptions 
does not modify our conclusions) then the experimental bounds ( (3.3. that

$$
G_{N}^{\nu}<6.0 \times 10^{-3} G_{F}
$$

Comparing with (12.12) we find that in the $S U(2)_{L}$ symmetric limit new interactions cannot have a significant contribution to the DAR signal observed at LSND.

We shall now argue that $S U(2)_{L}$ breaking effects are in general small and therefore cannot

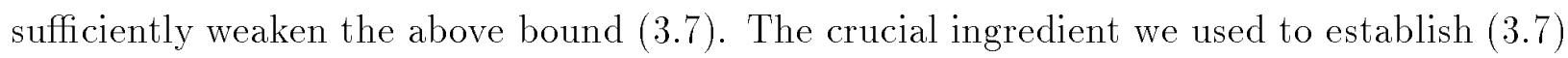
is $S U(2)_{L}$ invariance, i.e., we assumed that there is an $S U(2)_{L}$ rotation which transforms the four fermion operator that gives rise to $\mu^{+} \rightarrow e^{+} \bar{\nu}_{e} \nu_{\ell}$ to the one where the neutrinos are replaced by their charged lepton partners. If $S U(2)_{L}$ is an exact symmetry, then the coefficient of both operators coincide (up to a Clebsch-Gordon factor). While this relation is exact only when $S U(2)_{L}$ is unbroken, from electroweak precision data it follows that the breaking is small. As we will discuss in much detail, in the underlying theory the two related operators are induced by the exchange of heavy particles, that are members of one $S U(2)_{L}$ multiplet. If the intermediate particle is a singlet, or if the two processes are mediated by the same member of the multiplet, then $G_{N}^{\nu}=C_{C G} G_{N}^{\ell}\left(C_{C G}\right.$ is the Clebsch-Gordon factor). If not, then the equality is violated and the ratio of couplings is given by

$$
\frac{G_{N}^{\nu}}{G_{N}^{\ell}}=C_{C G} \frac{M_{1}^{2}}{M_{2}^{2}},
$$

where $M_{1}$ and $M_{2}$ are the masses of the particles belonging to the $S U(2)_{L}$ multiplet that mediate the processes described by $G_{N}^{\ell}$ and $G_{N}^{\nu}$, respectively. Then, if $M_{1} \neq M_{2}$ this multiplet will contribute to the $\rho$ parameter. Thus, we can use the bound on $\rho-1$ from the electroweak precision measurements, to determine the maximal ratio in eq. (13.8).

The contribution to the $\rho$ parameter depends on the Lorentz and $S U(2)_{L}$ representation of the multiplet. In general, higher dimensional representations contribute more. Therefore, it is sufficient to examine the case of a scalar $S U(2)_{L}$ doublet, where the maximal mass splitting can occur. From the recent data one finds that at $90 \%$ C.L. [120]

$$
\Delta M^{2} \equiv\left|M_{1}^{2}-M_{2}^{2}\right|<(77 \mathrm{GeV})^{2}
$$

The mass of the lightest component of any (non-singlet) multiplet is known to be more than $m_{Z} / 2$ from the measurement of the $Z$ width. Therefore, the largest possible effect arises for $M_{2}=45 \mathrm{GeV}$. Then, eq. ( $\left.{ }^{3} . \overline{9}_{1}^{\prime}\right)$ implies that $M_{1}<90 \mathrm{GeV}$ and we conclude that

$$
\frac{G_{N}^{\nu}}{G_{N}^{\ell}}=\frac{M_{1}^{2}}{M_{2}^{2}}<4.0
$$

We remark that this is a very conservative estimate for the maximal value of $M_{1}^{2} / M_{2}^{2}$, which could probably be improved by more rigorous arguments. Still, it is sufficient to show that 
the relaxation of the bound $\left(\begin{array}{l}\left(\overline{3} . \bar{Z}_{I}\right) \\ )\end{array}\right)$ ue to $S U(2)_{L}$ breaking effects could be at most a factor of four leading to

$$
G_{N}^{\nu}<2.4 \times 10^{-2} G_{F}, \quad r<5.8 \times 10^{-4}
$$

Thus, comparing with $\left(2 . \overline{1} 2_{1}^{\prime}\right)$ which requires $r>1.6 \times 10^{-3}$ we learn that the anomalous muon decays $\mu^{+} \rightarrow e^{+} \bar{\nu}_{e} \nu_{\ell}$ cannot significantly contribute to the LSND DAR result even for maximal $S U(2)_{L}$ breaking.

\section{SPECIFIC MODELS}

In this section we study the mechanism by which heavy intermediate particles can induce the new four-Fermi interactions. To be specific, we shall first introduce the general idea within two well-known extensions of the Standard Model and postpone a model-independent discussion until the next section.

First, we consider the minimal left-right symmetric model (LRSM) [21]. The relevant ingredient for our discussion is the existence of a Higgs triplet, $\Delta_{L}$, with the following lepton flavor violating couplings [is $2 \overline{2}]$

$$
\mathcal{H}_{\Delta}=i \sum_{\alpha, \beta=e, \mu, \tau} f_{\alpha \beta}\left(L_{\alpha L}^{T} C \tau_{2} \Delta_{L} L_{\beta L}\right)+\text { h.c. },
$$

where $L_{\alpha}$ denotes lepton doublet, $C$ is the charge conjugation matrix and

$$
\Delta_{L}=\left(\begin{array}{cc}
\Delta_{L}^{+} / \sqrt{2} & \Delta_{L}^{++} \\
\Delta_{L}^{0} & -\Delta_{L}^{+} / \sqrt{2}
\end{array}\right) .
$$

$\Delta_{L}^{+}$exchange leads to the effective four fermion interaction in eq. (12.5) with [-

$$
\left|G_{N}^{\nu_{\mu}}\right|=\frac{\left|f_{e e} f_{\mu \mu}^{*}\right|}{2 \sqrt{2} M^{2}\left(\Delta_{L}^{+}\right)}, \quad\left|G_{N}^{\nu_{\ell}}\right|=\frac{\left|f_{e e} f_{\mu \ell}^{*}\right|}{4 \sqrt{2} M^{2}\left(\Delta_{L}^{+}\right)},
$$

where in this case $\ell=e, \tau$ only. On the other hand, $\Delta_{L}^{++}$exchange leads to the related interaction involving four charged fermions, that we discussed in Section III I. The effective

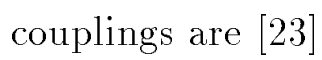

$$
\left|G_{N}^{\ell}\right|=\frac{\left|f_{e e} f_{\mu \ell}^{*}\right|}{4 \sqrt{2} M^{2}\left(\Delta_{L}^{++}\right)} .
$$

The diagrams that induce $G_{N}^{\nu_{\ell}}$ and $G_{N}^{\ell}$ are shown in Figs. ${ }_{-1}^{1} \operatorname{lin}_{0}^{1}$ for $\ell=e, \mu, \tau$, respectively. Provided that the mixing of $\Delta_{L}$ with other Higgs fields can be neglected, the triplet masses are related via [203ㄴ $M^{2}\left(\Delta_{L}^{++}\right)+M^{2}\left(\Delta_{L}^{0}\right)=2 M^{2}\left(\Delta_{L}^{+}\right)$implying that $M^{2}\left(\Delta_{L}^{++}\right) / M^{2}\left(\Delta_{L}^{+}\right) \leq 2$.

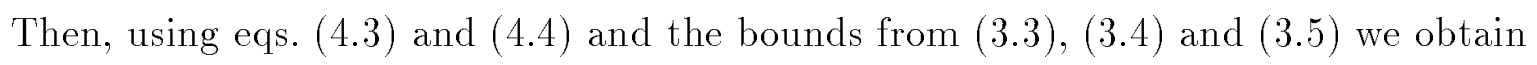

$$
r_{\text {LRS }}<1.4 \times 10^{-4}
$$


Thus, even for maximal mass-splitting, within LRSMs $\mu^{+} \rightarrow e^{+} \bar{\nu}_{e} \nu_{\ell}$ does not affect any of the existing terrestrial experiments and, in particular, cannot explain the LSND DAR-result.

The second example is a supersymmetric extension of the Standard Model without $R-$ parity [25] $[25]$. The trilinear $L L \bar{E}$ coupling between the leptonic chiral superfields $L$ and $E$ allows lepton flavor violating interactions which are mediated by sleptons. The relevant couplings are given by [206]

$$
\mathcal{H}_{R_{p}}=\frac{\lambda_{\imath \jmath \kappa}}{2}\left[\tilde{\nu}_{L}^{\imath} \ell_{L}^{3} \bar{\ell}_{R}^{\kappa}+\nu_{L}^{\imath} \tilde{\ell}_{L}^{3} \bar{\ell}_{R}^{\kappa}+\nu_{R}^{\imath c} \ell_{L}^{3} \tilde{\ell}_{R}^{\kappa *}-(\imath \rightarrow \jmath)\right]+\text { h.c. }
$$

where $\tilde{\nu}_{L}^{2}$ and $\tilde{\ell}_{L}^{2}$ are, respectively, the scalar neutrino and the (left-handed) scalar lepton fields of generation $\imath$, and the charge-conjugate fields are defined by $\nu_{R}^{\imath c}=C \bar{\nu}_{L}^{2 T}$. In this model $\mu^{+} \rightarrow e^{+} \bar{\nu}_{e} \nu_{\ell}$ proceeds via $\tilde{\ell}_{L}^{\prime}$ exchange, where $\ell^{\prime}=\tau(\mu)$ for $\ell=e, \mu(\tau)$, with effective coupling

$$
\left|G_{N}^{\nu \ell}\right|=\frac{\left|\lambda_{1 \ell^{\prime} 2} \lambda_{\ell \ell^{\prime} 1}^{*}\right|}{4 \sqrt{2} M^{2}\left(\tilde{\ell}_{L}^{\prime}\right)} .
$$

On the other hand, the charged lepton processes are mediated by $\tilde{\nu}_{\tau}\left(\tilde{\nu}_{\mu}\right)$ for $\ell=e, \mu(\tau)$ with

$$
\left|G_{N}^{\ell}\right|=\frac{\left|\lambda_{1 \ell^{\prime} 2} \lambda_{\ell \ell^{\prime} 1}^{*}\right|}{4 \sqrt{2} M^{2}\left(\tilde{\nu}_{\ell^{\prime}}\right)} .
$$

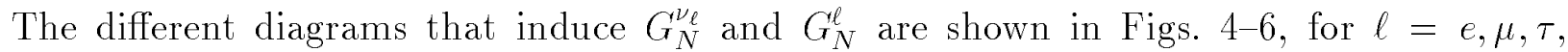
respectively. We find that $G_{N}^{\nu_{\ell}} / G_{N}^{\ell}=M^{2}\left(\tilde{\ell}_{L}^{\prime}\right) / M^{2}\left(\tilde{\nu}_{\ell^{\prime}}\right)$. The sleptons masses are related

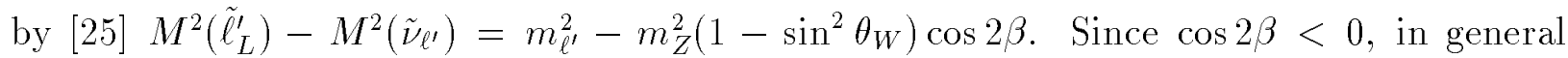
$M^{2}\left(\tilde{\ell}_{L}^{\prime}\right)>M^{2}\left(\tilde{\nu}_{\ell^{\prime}}\right)$. Therefore, within SUSY without $R$-parity a possible mass splitting would only strengthen the $S U(2)_{L}$ symmetric bound given by

$$
r_{\mathbb{R}_{\mathrm{P}}} \leq 9 \times 10^{-6}
$$

Obviously this is much too small to affect any of the existing terrestrial experiments and below the range (2.11 $)$ needed to explain the LSND result. As we shall see in the next section the two explicit examples we presented here in fact exhaust all the possible purely leptonic couplings induced by intermediate scalar particles.

\section{MODEL INDEPENDENT ANALYSIS}

We have seen in the previous section within two explicit models the tight relation between the operators that induce $\mu^{+} \rightarrow e^{+} \bar{\nu}_{e} \nu_{\ell}$ and those where the neutrinos are replaced by their charged lepton partners. In this section we show in a model independent way that in our case it is impossible to evade the close relation between these operators. 
The exchange of a (heavy) boson between two fermion bilinears induces a four-fermion operator whose effective coupling at low energies depends only on the boson mass and the elementary (trilinear) couplings. To obtain the most general set of such operators for New Physics in the leptonic sector only, let us write all trilinear couplings involving at least one doublet $L$ (which contains the required neutrino) and at most one singlet $E$ (which contains a right-handed charged lepton) and the respective antiparticles [는. A priori there are only four such bilinears to which the intermediate particle can couple. They are tabulated in Tab. 1 together with their $S U(2)_{L}$ representations and the possible values for the charge $Q$ and the hypercharge $Y$ (without loss of generality we suppress the complex conjugated bilinears which have opposite $Y, Q)$.

Tab. 1: Lepton-Lepton Bilinears

\begin{tabular}{|c|c|c|c|c|}
\hline Bilinear & Coupling & $S U(2)_{L}$ & $Q$ & $Y$ \\
\hline \hline$L L$ & scalar & 1,3 & $0,-1,-2$ & -1 \\
\hline $\bar{E} L$ & scalar & 2 & 1,0 & $1 / 2$ \\
\hline $\bar{L} L$ & vector & 1,3 & $1,0,-1$ & 0 \\
\hline$E L$ & vector & 2 & $-1,-2$ & $-3 / 2$ \\
\hline
\end{tabular}

Due to the conservation of $Y$ one can only construct operators that result from the coupling between any of these bilinears and its complex conjugate. So there are only six possibilities, which we will discuss one by one: An intermediate scalar singlet cannot contribute to $\mu^{+} \rightarrow e^{+} \bar{\nu}_{e} \nu_{\ell}$. The reason is that the final state of this muon decay has to contain an $e^{+}$and a $\bar{\nu}_{e}$. Since the $L L$ bilinear has to form an $S U(2)_{L}$ singlet, it has to be antisymmetric in flavor space. This implies that one cannot produce an $e^{+}$and a $\bar{\nu}_{e}$ simultaneously by exchanging a charged scalar singlet. (Note that e.g. $\mu^{+} \rightarrow e^{+} \bar{\nu}_{\tau} \nu_{e}$ could be mediated by a scalar singlet, but that the effective operator responsible for this process cannot be related by an $S U(2)_{L}$ rotation to the one where the neutrinos are replaced by their charged lepton partners.) The two remaining possibilities that involve intermediate scalar particles are those that appeared within the two specific models that we discussed in the previous section, i.e., the triplet $\Delta_{L}$ in LRSMs and the doublet $L^{T}=\left(\tilde{\nu}_{\ell^{\prime}}, \tilde{\ell}^{\prime}\right)$ in SUSY without $R$-parity. We only used model-specific ingredients to explain why in these models the mass splitting is always smaller than the maximally allowed. Still, it can be easily checked that even for maximal splitting the possible effect is too small. We therefore conclude that scalar particles in general cannot mediate $\mu^{+} \rightarrow e^{+} \bar{\nu}_{e} \nu_{\ell}$ at a rate required to explain the DAR-result of LSND.

The remaining entries in the Tab. 1 require an intermediate spin-1 boson with vector couplings. An $S U(2)_{L}$ singlet couples to $\left(\bar{L} L^{\prime}\right)_{s}=\overline{\nu_{\ell}} \gamma_{\mu} \nu_{\ell^{\prime}}+\overline{\ell_{L}} \gamma_{\mu} \ell_{L}^{\prime}$. This implies that the couplings for any interaction mediated by a singlet remain exactly the same when exchanging the two neutrinos by their charged $S U(2)_{L}$ partners. Therefore we can directly apply 


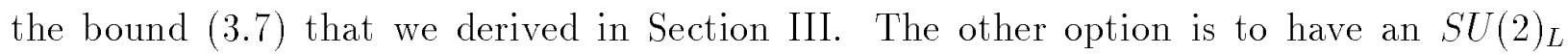
triplet $W^{\prime \mu}$ that couples to $\bar{L} \gamma_{\mu} \tau L$ just like the Standard Model vector-boson $W$. If we allow for flavor off-diagonal couplings $g_{\alpha \beta}$ the exchange of the charged components $\left(W^{ \pm}\right)$ induces $\left(\overline{\nu_{e}} \gamma_{\mu} \mu_{L}\right)\left(\overline{e_{L}} \gamma^{\mu} \nu_{\ell}\right)$ and the exchange of the neutral component $\left(Z^{\prime}\right)$ gives rise to $\left(\overline{e_{L}} \gamma_{\mu} \mu_{L}\right)\left(\overline{e_{L}} \gamma^{\mu} \ell_{L}\right)$. For both operators the effective couplings are proportional to $g_{e \mu} g_{e \ell}^{*}$ and differ only by the mass splitting between the $W^{\prime \pm}$ and $Z^{\prime}$. Thus we find again that the muon decay $\mu^{+} \rightarrow e^{+} \bar{\nu}_{e} \nu_{\ell}$ is tightly related to the charged lepton decays or muonium-antimuonium conversion. Finally the $E L$ bilinear requires a spin-1 vector doublet with $Y=3 / 2$. In this case a rotation between $\ell$ and $\nu_{\ell}$ goes along with the exchange of the two components of this vector doublet, so the $S U(2)_{L}$ symmetry guarantees that $\mu^{+} \rightarrow e^{+} \bar{\nu}_{e} \nu_{\ell}$ and the respective charged lepton processes have the same couplings up to the mass-splitting between the two members of the vector doublet which is small.

We thus conclude that any purely leptonic process, that conserves total lepton number, cannot contribute significantly to the LSND DAR-signal.

\section{SEMI-LEPTONIC INTERACTIONS}

So far we have restricted our analysis to the case of having New Physics only in $\mu^{+} \rightarrow e^{+} \bar{\nu}_{e} \nu_{\ell}$ showing that its rate cannot be sufficient to provide $\bar{\nu}_{e}$ 's at a rate seen at LSND. While this is a reasonable assumption for LRSMs, where the intermediate particles that induce the new interactions only couple to leptons, in general also new semi-leptonic interactions can play a role.

In fact, for LSND the production reaction for the DIF $\left(\pi^{+} \rightarrow \mu^{+} \nu\right)$ and the detection reaction from both the $\operatorname{DIF}\left(\nu n \rightarrow p e^{-}\right)$and the $\operatorname{DAR}\left(\bar{\nu} p \rightarrow n e^{+}\right)$are semi-leptonic. All the semi-leptonic four-Fermi operators of relevance to LSND involve a $u$ and a $d$-quark, a charged lepton and only one neutrino. While the involved quarks necessarily belong to the first generation and the charged leptons must be either the muon or the electron, a priori all the three neutrino flavors could be involved in the New Physics contribution to the semi-leptonic reactions.

The four-Fermi operators that are relevant for the detection reactions are of the form $\left(\nu_{\ell} e^{+} d \bar{u}\right)$ and for the DIF production the relevant operator is $\left(\nu_{\ell} \mu^{+} d \bar{u}\right)$. Applying similar arguments as in our discussion of the purely leptonic new interactions, one can use the $S U(2)_{L}$ symmetry to relate these operators to the ones where the neutrino is replaced by its charged lepton partner, namely

$$
\begin{aligned}
& \left(\nu_{\ell} e^{+} d \bar{u}\right) \stackrel{S U(2)}{\Longleftrightarrow} L\left(\ell^{-} e^{+} q \bar{q}\right) \\
& \left(\nu_{\ell} \mu^{+} d \bar{u}\right) \stackrel{S U(2) L}{\Longleftrightarrow}\left(\ell^{-} \mu^{+} q \bar{q}\right),
\end{aligned}
$$

where $q=u, d$.

Let us ignore for the moment $S U(2)_{L}$ breaking effects and the Dirac structure that we

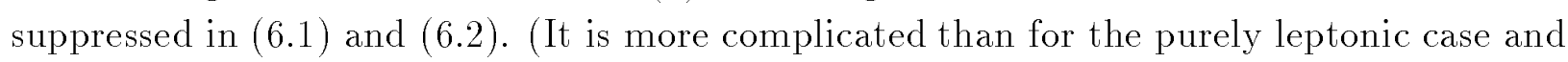


we will discuss how these operators arise and relate at the elementary level later on.) Then the upper bounds on processes which would be induced by the operators that contain only charged particles can be used to put stringent constraints on the semi-leptonic reactions relevant to LSND.

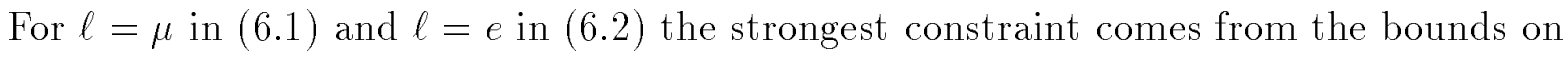
muon conversion on nuclei [riv]

$$
\frac{\sigma\left(\mu^{-\mathrm{T} i} \rightarrow e^{-\mathrm{T} i}\right)}{\sigma\left(\text { all } \mu^{-\mathrm{T} i} \text { capture }\right)}<4.3 \times 10^{-12} \text {. }
$$

For the effective coupling of the $\left(\bar{\mu}_{L} \gamma_{\mu} e_{L}^{+} q_{L} \gamma^{\mu} \bar{q}_{L}\right)$ operator this implies

$$
G_{N}(\mu e q q)<2.1 \times 10^{-6} G_{F},
$$

which is four orders of magnitudes smaller than the coupling in (25.12in $)$ needed to produce a signal for LSND. We note that the above bound (16.4) could be somewhat relaxed due to differences in the matrix elements $\left[\begin{array}{c}\overline{2} \\ \underline{2}\end{array}\right]$, different Dirac structure and $S U(2)_{L}$ breaking effects which we ignored. Still, assuming that there are no fine-tuned cancellations, it is safe to conclude that the coupling of semi-leptonic reactions which violate only the $L_{e}$ and $L_{\mu}$ lepton family numbers are much too small to be relevant for LSND.

If the $\nu_{\tau}$ is involved then all the three lepton family numbers are violated and new interactions are required for both the production and detection processes. In this case the

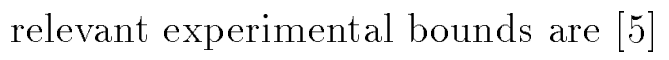

$$
\mathrm{BR}\left(\tau \rightarrow e \pi^{0}\right)<3.7 \times 10^{-6}, \quad \mathrm{BR}\left(\tau \rightarrow \mu \pi^{0}\right)<4.0 \times 10^{-6} .
$$

Normalizing these branching ratios to $\operatorname{BR}\left(\tau^{-} \rightarrow \pi^{-} \nu_{\tau}\right)=0.11$ and using isospin symmetry we find that the coupling of the operator $\left(\bar{\tau}_{L} \gamma_{\mu} \ell_{L}^{+} q_{L} \gamma^{\mu} \bar{q}_{L}\right)$, satisfies the constraint

$$
G_{N}(\tau \ell q q)<8.5 \times 10^{-3} G_{F}
$$

for $\ell=e, \mu$. For the DIF, $S U(2)_{L}$ relates this coupling to those describing the production $(\ell=\mu)$ and the detection $(\ell=e)$ process. For the DAR, the production must be $\mu^{+} \rightarrow e^{+} \bar{\nu}_{\tau} \nu_{\ell}$. Using the agreement between the tau lifetime and its purely leptonic decay width, one can conclude that $\operatorname{BR}\left(\mu^{+} \rightarrow e^{+} \bar{\nu}_{\tau} \nu_{\ell}\right)<5 \times 10^{-3}$. Therefore, complications that arise from isospin breaking effects, possible different Dirac structure and $S U(2)_{L}$ breaking effects can be safely ignored also in this case. We conclude that the constraints arising from muon conversion on nuclei and $\tau \rightarrow \ell \pi^{0}$ exclude new semi-leptonic interactions from significantly affecting either of the two LSND results.

We turn now to a model independent analysis of the possible couplings and their relations for the semi-leptonic channels using similar arguments as in Section IV- Our goal is to show explicitly that it is impossible to evade the tight relation between operators related by $S U(2)_{L}$ rotations, which is crucial for the arguments presented above to be valid in general. 
Consider first the bilinears that consist of two quarks. In order to couple to the leptonic bilinears of Tab. 1 they must be $S U(3)_{C}$ singlets. Hence they contain one quark $Q, D$ or $U$ and one anti-quark, where $Q$ is the doublet and $D$ and $U$ are $S U(2)_{L}$ singlets. They are summarized in Tab. 2.

Tab. 2: Quark-Quark Bilinears

\begin{tabular}{|c|c|c|c|c|}
\hline Bilinear & Coupling & $S U(2)_{L}$ & $Q$ & $Y$ \\
\hline \hline $\bar{U} Q$ & scalar & 2 & $0,-1$ & $-1 / 2$ \\
\hline $\bar{D} Q$ & scalar & 2 & 1,0 & $1 / 2$ \\
\hline $\bar{Q} Q$ & vector & 3,1 & $1,0,-1$ & 0 \\
\hline $\bar{U} D$ & vector & 1 & -1 & -1 \\
\hline $\bar{U} U$ & vector & 1 & 0 & 0 \\
\hline $\bar{D} D$ & vector & 1 & 0 & 0 \\
\hline
\end{tabular}

Due to the conservation of $Y$ it follows that from the singlet-singlet bilinears (the last three entries in Tab. 2) only $\bar{U} U$ and $\bar{D} D$ couple to the vector singlet of $\bar{L} L$ of Tab. 1 . However the resulting four fermion operators do not contribute to the semi-leptonic reactions of interest since they cannot change the charge of the involved leptons and quarks.

The $\bar{U} Q$ and $\bar{D} Q$ bilinears couple via a scalar $S U(2)_{L} \operatorname{doublet}($ with $Y= \pm 1 / 2$ ) to $\bar{E} L$ (or its complex conjugate). Let us use here the familiar notation from SUSY without $R$-parity for the couplings and the scalar particles (none of our arguments requires supersymmetry and therefore the underlying theory providing the new couplings is arbitrary). The coupling $\lambda_{\imath \jmath \kappa}^{\prime} L_{L}^{\imath} Q_{L}^{\jmath}{\overline{D_{R}}}^{\kappa}$ between the chiral superfields $L_{L}, Q_{L}$ and $D_{R}$ induces exactly the required coupling between the quark bilinear and the scalar doublet

$$
\lambda_{\imath \jmath \kappa}^{\prime}\left[\tilde{\nu}_{L}^{2} d_{L}^{\jmath}{\overline{d_{R}}}^{\kappa}+\tilde{\ell}_{L}^{2} u_{L}^{3}{\overline{d_{R}}}^{\kappa}\right]+\text { h.c. . }
$$

The coupling of the scalar doublet to the lepton bilinear proceeds via the appropriate term

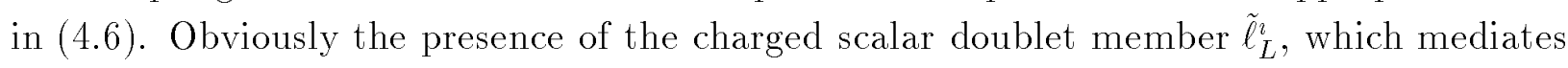
the semi-leptonic processes relevant to LSND, generically requires the presence of its neutral doublet partner $\tilde{\nu}_{L}^{2}$. Then the effective couplings for the operator $\left(\overline{u_{L}} d_{R}\right)\left(\overline{\ell_{R}^{\prime}} \nu_{\ell}\right)\left(\ell \neq \ell^{\prime}\right)$ coincides with the effective couplings for the operator $\left(\overline{d_{L}} d_{R}\right)\left(\overline{\ell_{R}^{\prime}} \ell_{L}\right)$ up to the mass splitting between $\tilde{\ell}_{L}^{2}$ and $\tilde{\nu}_{L}^{2}$. However, as we have shown in the beginning of this section this operator is severely constrained and thus cannot significantly contribute to semi-leptonic processes that change lepton flavor. So we conclude that an intermediate scalar doublet cannot contribute significantly to the LSND results.

The remaining candidate for a coupling to a leptonic bilinear is the $\bar{Q} Q$. In this case the intermediate particle must be either a spin-1 triplet or singlet with $Y=0$. Since the singlet is neutral it cannot mediate the charged-current semi-leptonic processes that we are interested in. For the triplet we require a vector boson $W^{\prime}$ that has both flavor diagonal 
couplings to quarks and flavor off-diagonal couplings $g_{\ell \ell^{\prime}}$ to leptons (like the one we evoked for the self-coupling of the $\bar{L} L$ bilinear). Then the exchange of the charged components $\left(W^{ \pm}\right)$induces $\left(\overline{u_{L}} \gamma_{\mu} d_{L}\right)\left(\overline{\ell_{L}} \gamma^{\mu} \nu_{\ell^{\prime}}\right)$ and the exchange of the neutral component $\left(Z^{\prime}\right)$ gives rise to $\left(\overline{q_{L}} \gamma_{\mu} q_{L}\right)\left(\overline{\ell_{L}} \gamma^{\mu} \ell_{L}^{\prime}\right)$. For both operators the effective couplings are proportional to $g_{\ell \ell^{\prime}}$ and differ only by the mass splitting between the $W^{\prime \pm}$ and $Z^{\prime}$. Thus the argument using related processes containing only charged fermions that we presented in the beginning works equally well for an intermediate spin-1 boson.

Having exhausted the quark-quark bilinears we now turn to the possibility of having

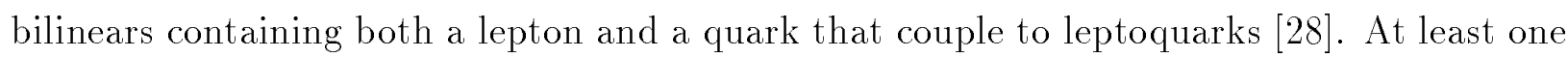
bilinear must contain the doublet $L$ (since we require a neutrino) and any of the quark fields $Q, D$ and $U$ leading to the following combinations:

Tab. 3a: Quark-Lepton $L$ Bilinears

\begin{tabular}{|c|c|c|c|c|}
\hline Bilinear & Coupling & $S U(2)_{L}$ & $Q$ & $Y$ \\
\hline \hline$Q L$ & scalar & 1,3 & $2 / 3,-1 / 3,-4 / 3$ & $-1 / 3$ \\
\hline $\bar{D} L$ & scalar & 2 & $1 / 3,-2 / 3$ & $-1 / 6$ \\
\hline $\bar{U} L$ & scalar & 2 & $-2 / 3,-5 / 3$ & $-7 / 6$ \\
\hline $\bar{Q} L$ & vector & 1,3 & $1 / 3,-2 / 3,-5 / 3$ & $-2 / 3$ \\
\hline$D L$ & vector & 2 & $-1 / 3,-4 / 3$ & $-5 / 6$ \\
\hline$U L$ & vector & 2 & $2 / 3,-1 / 3$ & $1 / 6$ \\
\hline
\end{tabular}

Let us first consider those four-Fermi operators that are built only from the bilinears of Tab. 3a. The first three bilinears in Tab. 3a require scalar couplings, while the other three can only couple to a spin-1 particle. The conservation of angular momentum forbids that bilinears that have a different type of couplings couple to each other. Then using the conservation of hypercharge one can see that the allowed four-fermion operators only arise from bilinears that couple to themselves. It follows that operators from bilinears with a singlet quark always contain either the $D$ or the $U$ singlet quarks, but not both, and are therefore of no relevance to the semi-leptonic reactions that could explain LSND.

The $Q L$ bilinear could couple either to a scalar singlet or triplet of $S U(2)_{L}$. The singlet coupling involves the term $u_{L} \ell_{L}-d_{L} \nu_{\ell}$ implying that a vertex where the scalar singlet couples to the neutrino and the $d$-quark has the same coupling strength as to the charged lepton partner and the $u$-quark. Thus the operator $\left(\overline{u_{L}} \overline{\ell_{L}}\right)\left(d_{L} \nu_{\ell^{\prime}}\right)\left(\ell \neq \ell^{\prime}\right)$ has the same coupling as $\left(\overline{u_{L}} \overline{\ell_{L}}\right)\left(u_{L} \ell_{L}^{\prime}\right)$ and we can again apply our argument using the bounds on $\mu \mathrm{Ti} \rightarrow e \mathrm{Ti}$ and $\tau \rightarrow \ell \pi^{0}$. Similarly, when the $Q L$ bilinear forms an $S U(2)_{L}$ triplet it is the $Q=1 / 3$ component that is relevant which couples to $u_{L} \ell_{L}+d_{L} \nu_{\ell}$. Again, the same arguments as for the singlet case apply, and the related charged lepton processes put severe bounds on this case as well.

Thus the only remaining candidate is the $\bar{Q} L$ bilinear. The intermediate leptoquark $X$ must be a spin-1 boson. It could be a $S U(2)_{L}$ singlet with $Q=Y=2 / 3$ that induces the 
operator $\left(\overline{\nu_{\ell}} \gamma_{\mu} u_{L}+\overline{\ell_{L}} \gamma_{\mu} d_{L}\right)\left(\overline{u_{L}} \gamma^{\mu} \nu_{\ell^{\prime}}+\overline{d_{L}} \gamma^{\mu} \ell_{L}^{\prime}\right)$, which obviously gives the same couplings for the $S U(2)_{L}$ related processes that we study. An intermediate spin- 1 triplet $X^{\mu}$ with $Y=2 / 3$ couples to $\bar{Q} \gamma_{\mu} \tau L$. The relevant coupling for our discussion is induced by the $Q=2 / 3$ component of $X_{\mu}$ which couples via $\tau_{3}$ to the fermions $\sum_{\ell=e, \mu, \tau} h_{\ell} X_{\mu}^{(2 / 3)}\left[\overline{u_{L}} \gamma^{\mu} \nu_{\ell}-\overline{d_{L}} \gamma^{\mu} \ell_{L}\right]$. Hence - no surprise - the operator $\left(\overline{u_{L}} \gamma_{\mu} \nu_{\ell}\right)\left(d_{L} \gamma^{\mu} \overline{\ell_{L}^{\prime}}\right)$ has the same effective coupling as $\left(\overline{d_{L}} \gamma_{\mu} \ell_{L}\right)\left(d_{L} \gamma^{\mu} \overline{\ell_{L}^{\prime}}\right)$ and the discussed $S U(2)_{L}$ symmetry also works for this case.

Finally, we have to consider the case when the bilinears from Tab. 3a do not couple to themselves but to those containing a lepton singlet $E$ and a quark field. The possible bilinears are given in Tab. $3 \mathrm{~b}$.

Tab. 3b: Quark-Lepton E Bilinears

\begin{tabular}{|c|c|c|c|c|}
\hline Bilinear & Coupling & $S U(2)_{L}$ & $Q$ & $Y$ \\
\hline \hline $\bar{Q} E$ & scalar & 2 & $-2 / 3,-5 / 3$ & $-7 / 6$ \\
\hline$D E$ & scalar & 1 & $-4 / 3$ & $-4 / 3$ \\
\hline$U E$ & scalar & 1 & $-1 / 3$ & $-1 / 3$ \\
\hline$Q E$ & vector & 2 & $-1 / 3,-4 / 3$ & $-5 / 6$ \\
\hline $\bar{D} E$ & vector & 1 & $-2 / 3$ & $-2 / 3$ \\
\hline $\bar{U} E$ & vector & 1 & $-5 / 3$ & $-5 / 3$ \\
\hline
\end{tabular}

Comparing the entries for the bilinears in Tab. 3a and Tab. $3 \mathrm{~b}$ we find that there are four possibilities: $\bar{Q} E$ and $U \bar{L}$ via a scalar doublet, $U E$ and $\bar{Q} \bar{L}$ via a scalar singlet, $Q E$ and $\bar{D} \bar{L}$ via a vector doublet and $\bar{D} E$ and $Q \bar{L}$ via a vector singlet. Repeating similar arguments as presented before one can show that in all of these cases also the corresponding charge lepton operators are induced.

We thus conclude that lepton number conserving semi-leptonic processes cannot contribute significantly to any of the LSND signals.

\section{IMPLICATIONS AND CONCLUSIONS}

Extensions of the Standard Model in general do not conserve individual lepton numbers and therefore provide an alternative mechanism for neutrino flavor conversion that may show up in neutrino oscillation experiments. We have argued that the experimental constraints on such lepton flavor violating interaction do not allow such an interpretation for any of the LSND results. Our argument relies on the bounds from $S U(2)_{L}$ related reactions containing the charged partner of the relevant neutrino. We have shown explicitly the relations between the effective coupling of the two types of reactions within LRSMs and SUSY without $R$ parity as examples for New Physics that affects the anomalous muon decay. Moreover, we have demonstrated in a model-independent way that the ratio of these couplings is always of 
order one and that the deviation from unity is only due to a generically small mass splitting between the bosonic members of an $S U(2)_{L}$ multiplet and some Clebsch-Gordan coefficients.

It is still interesting to ask whether lepton flavor violation could influence other neutrino experiments and whether their explanation in terms of neutrino oscillation might be modified or even spoiled by the New Physics. Recall that both the solar and atmospheric neutrino experiments detect quite a large deviation from the predicted neutrino-flux by a factor $\sim 1 / 2$ with experimental uncertainties of about $10 \%$. In general the effects of New Physics on the production or detection process are much smaller and hence cannot influence those experiments drastically via those processes. However, if the MSW effect is the correct solution to the solar neutrino problem, then New Physics may influence the resonant conversion [20 if reactions of the type

$$
\nu_{e} f \rightarrow \nu_{\ell} f
$$

where $f=e, u, d$ and $\ell=\mu$ or $\tau$, are present. Note that while the process ( $\left.1 . \overline{1} \overline{1}_{1}^{\prime}\right)$ and the flavor violating semi-leptonic reactions that we discussed always violate the individual lepton numbers $L_{e}$ and $L_{\ell}$ by one unit, this is only true for the anomalous muon decay $\mu^{+} \rightarrow e^{+} \bar{\nu}_{e} \nu_{e}$. The two other decays producing $\nu_{\mu}$ or $\nu_{\tau}$ in the final state violate $L_{e}$ by two units. Hence the New Physics processes that are potentially relevant for short baseline experiments and the MSW mechanism are not necessarily related. But generically all types of reactions could be present. While saturating the current bounds on the effective couplings for reactions involving a $\nu_{\tau}$ is not sufficient to produce a significant effect for LSND (since they are suppressed both for the production and the detection), this is not true for solar

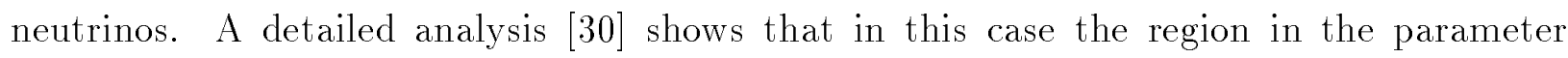
space that corresponds to the small mixing angle solution is somewhat shifted. The shift is basically in the value of the mixing angle, while the required mass-squared difference is almost unaltered by the presence of the New Physics. We note that also the effect of lepton flavor violating interactions on the resonant neutrino conversion in supernovae has been studied survival probability for a large region of parameter space.

We conclude that the presence of lepton flavor violating interactions cannot solve the problem of explaining the three observed $\Delta m^{2}$ scales with three neutrino generations. We did not study the possibility that lepton number violation processes may be relevant. For example, the decay $\mu^{+} \rightarrow e^{+} \bar{\nu}_{e} \bar{\nu}_{\ell}$ may explain the DAR LSND result [i 15.

\section{ACKNOWLEDGMENTS}

We thank Haim Goldberg, Yossi Nir, Damien Pierce, Tom Rizzo, Tom Weiler and Jim Wells for helpful discussions. Y.G. is supported by the U.S. Department of Energy under contract DE-AC03-76SF00515. 


\section{REFERENCES}

[1] Y. Fukuda et al., Kamiokande Collaboration, Phys. Lett. B335 (1994) 237;

R. Becker-Szendy et al., IMB Collaboration, Phys. Rev. Lett. 69 (1992) 1010;

W.W.M Allison et al., Soudan-2 Collaboration, Phys. Lett. B391 (1997) 491.

[2] For a recent analysis see e.g.:

J.N. Bahcall, P.I. Krastev and A.Yu. Smirnov, hep-ph 99807216

[3] Y. Fukuda et al., Super-Kamiokande Collaboration, hep-ex 29007003

[4] M. Apollonio et al., CHOOZ collaboration, Phys. Lett. B420 (1998) 397.

[5] C. Caso et al. [Particle Data Group], Eur. Phys. J. C3, 1 (1998).

[6] C. Athanassopoulos et al., LSND Collaboration, Phys. Rev. Lett. 77 (1996) 3082.

[7] C. Athanassopoulos et al., LSND Collaboration, Phys. Rev. Lett. 81 (1998) 1774.

[8] See e.g., J.T. Peltoniemi, hep-ph 49506228

S. Goswami, Phys. Rev. D55 (1997) 2931;

N. Okada and O. Yasuda, Int. J. Mod. Phys. A12 (1997) 3669;

S.M. Bilenky, C. Giunti and W. Grimus, Eur. Phys. J. C1 (1998) 247.

[9] Z.G. Berezhiani and R.N. Mohapatra, Phys. Rev. D52 (1995) 6607;

E. Ma and P. Roy, Phys. Rev. D52 (1995) 4780;

E. Ma, Phys. Lett. B380 (1996) 286; Mod. Phys. Lett. A 11 (1996) 1898;

E.J. Chun, A.S. Joshipura and A.Yu. Smirnov,

Phys. Lett. B357 (1995) 608; Phys. Rev. D54 (1996) 4654;

K. Benakli and A.Yu. Smirnov, Phys. Rev. Lett. 79 (1997) 4314;

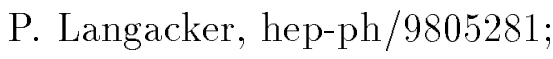

B. Brahmachari and R.N. Mohapatra, hep-ph

N. Arkani-Hamed and Y. Grossman, ihep-ph 29806223.

[10] V. Barger, S. Pakvasa, T.J. Weiler and K. Whisnant, hiep-ph $[9806328$

[11] C.Y. Cardall and G.M. Fuller, Phys. Rev. D53 (1996) 4421.

[12] A. Acker and S. Pakvasa, Phys. Lett. B397 (1997) 209.

[13] R.P. Thun and S. McKee, hep-ph

[14] G.L. Fogli, E. Lisi, A. Marrone, G. Scioscia, hep-ph

[15] P. Herczeg, proceedings of "beyond the desert 1997".

[16] L.M. Johnson and D.W. McKay, Phys. Lett. B433 (1998) 355.

[17] Y. Grossman, Phys. Lett. B359 (1995) 141. 
[18] See e.g., R.E. Marshak, Riazuddin and C.P. Ryan,

Theory of weak interactions in particle physics (Wiley, New York, 1969).

[19] K. Jungmann [for the PSI experiment], "thep-ex/9805014:

[20] D. Pierce, private communication.

[21] R.N. Mohapatra and G. Senjanović,

Phys. Rev. Lett. 44 (1980) 912; Phys. Rev. D23 (1981) 165.

[22] J.F. Gunion, J. Grifols and A. Méndez, B. Kayser and F. Olness, Phys. Rev. D40 (1989) 1546.

[23] P. Herczeg and R.N. Mohapatra, Phys. Rev. Lett. 69 (1992) 2475;

R.N. Mohapatra, Z. Phys. C56 (1992) S117.

[24] J.A. Coarasa, A. Méndez and J. Solà, Phys. Lett. B374 (1996) 131.

[25] See e.g., H.E. Haber, in Recent Directions in Particle Theory,

Proc. of the 1992 Theoretical Advanced Study Institute in Elementary Particle Physics,

J. Harvey and J. Polchinski, eds. (World Scientific, Singapore, 1993) p. 589.

[26] A. Halprin and and A. Masiero, Phys. Rev. D48 (1993) R2987.

[27] F. Cuypers and S. Davidson, Eur. Phys. J. C2 (1998) 503.

[28] S. Davidson, D. Bailey and B.A. Campbell, Z. Phys. C61 (1994) 613.

[29] M. Fukugita and T. Yanagida, Phys. Lett. B206 (1988) 93;

E. Roulet, Phys. Rev. D44 (1991) 935;

M.M. Guzzo, M. Masiero and S.T. Petcov, Phys. Lett. B260 (1991) 154;

V. Barger, R.J.N. Phillips and K. Whisnant, Phys. Rev. D44 (1991) 1629;

S. Degl'Innocenti and B. Ricci, Mod. Phys. Lett. A 8 (1993) 471.

[30] S. Bergmann, Nucl. Phys. B515 (1998) 363 (hep-ph [970739

[31] S.W. Mansour and T.K. Kuo, Phys. Rev. D58 (1998) 013012.

[32] S. Bergmann and A. Kagan, ihep-ph 


\section{FIGURES}

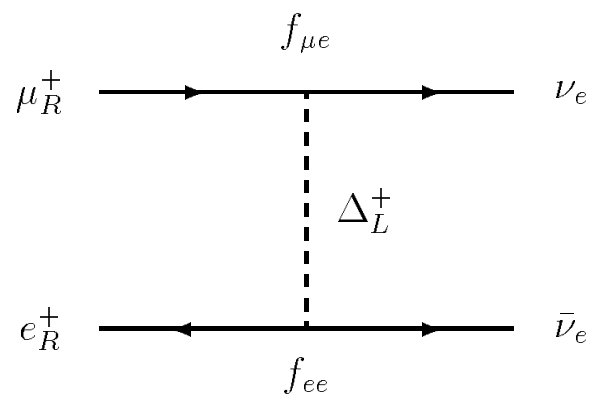

(a)

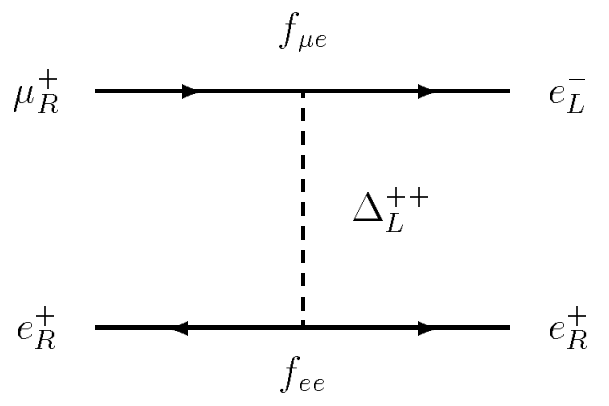

(b)

FIG. 1. Diagrams for (a) $\mu^{+} \rightarrow e^{+} \bar{\nu}_{e} \nu_{e}$ and (b) $\mu \rightarrow 3 e$ for LRSMs

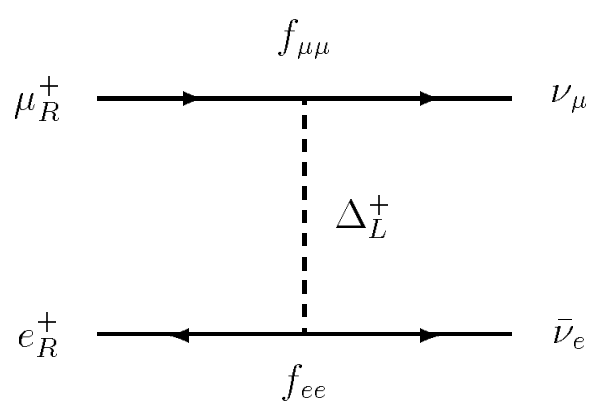

(a)

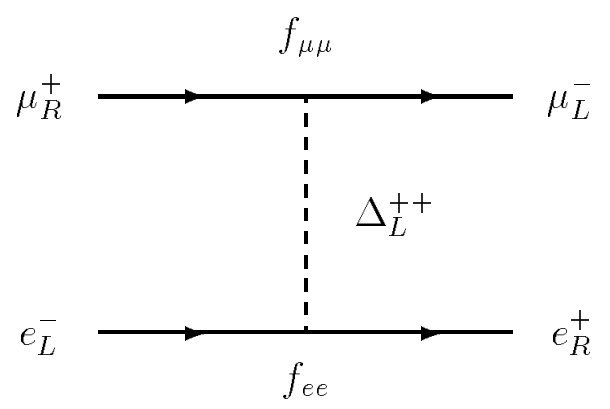

(b)

FIG. 2. Diagrams for (a) $\mu^{+} \rightarrow e^{+} \bar{\nu}_{e} \nu_{\mu}$ and (b) $\mu^{+} e^{-} \rightarrow \mu^{-} e^{+}$for LRSMs

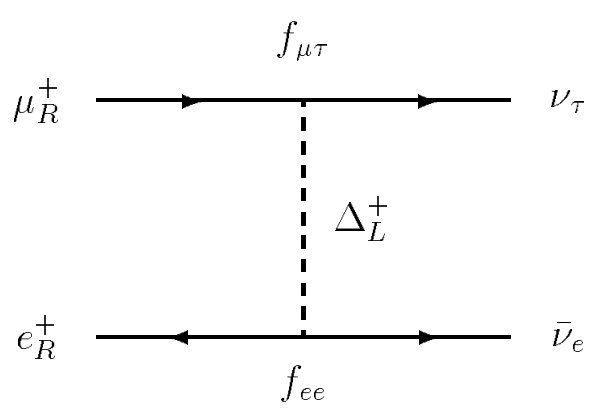

(a)

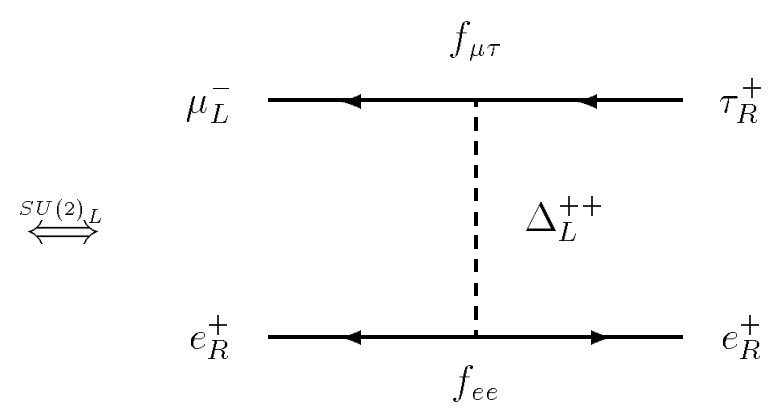

(b)

FIG. 3. Diagrams for (a) $\mu^{+} \rightarrow e^{+} \bar{\nu}_{e} \nu_{\tau}$ and (b) $\tau^{-} \rightarrow \mu^{+} e^{-} e^{-}$for LRSMs 

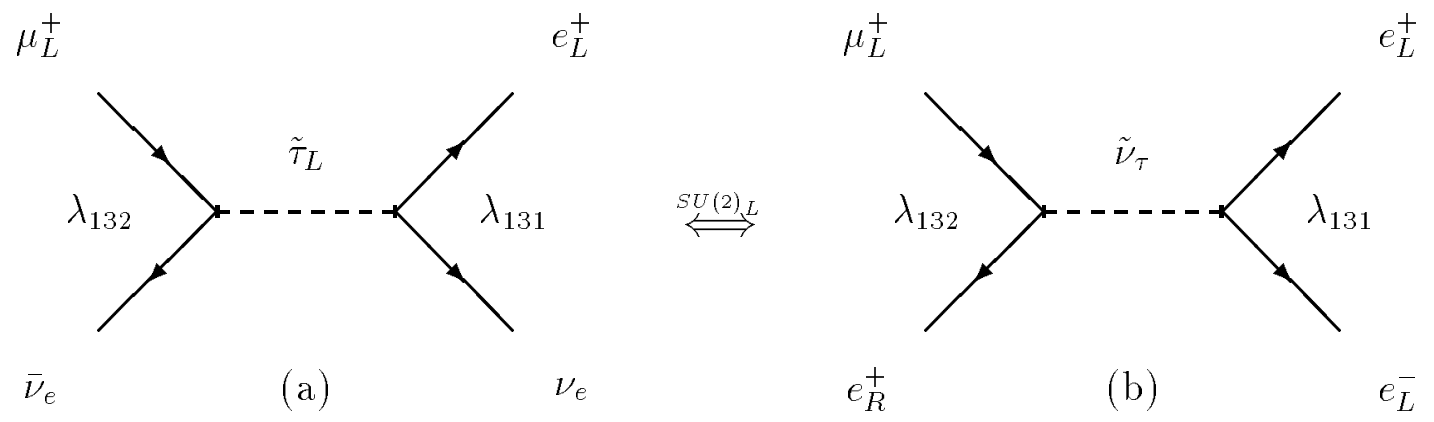

FIG. 4. Diagrams for (a) $\mu^{+} \rightarrow e^{+} \bar{\nu}_{e} \nu_{e}$ and (b) $\mu \rightarrow 3 e$ for SUSY without $R_{p}$

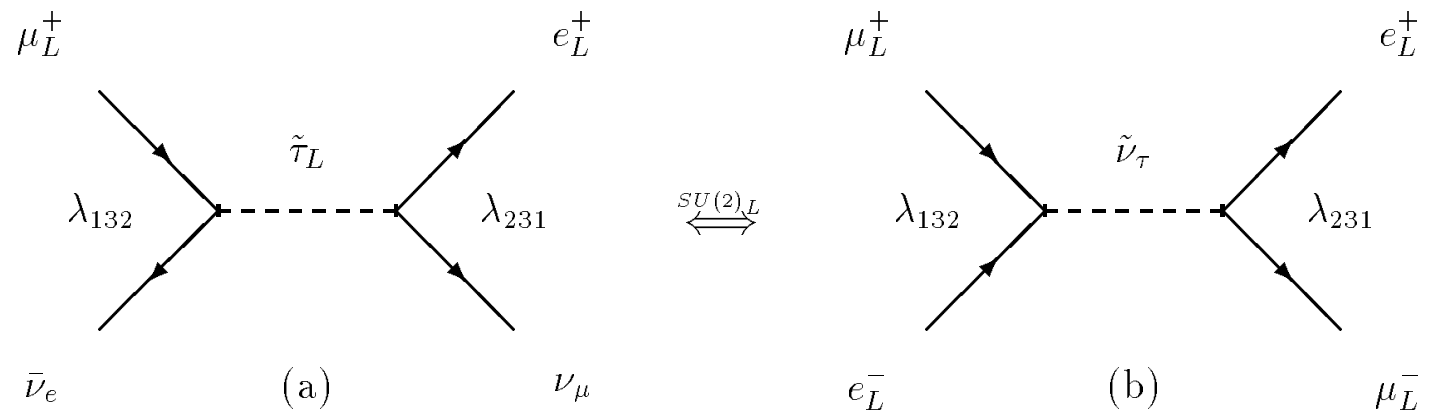

FIG. 5. Diagrams for (a) $\mu^{+} \rightarrow e^{+} \bar{\nu}_{e} \nu_{\mu}$ and (b) $\mu^{+} e^{-} \rightarrow \mu^{-} e^{+}$for SUSY without $R_{p}$

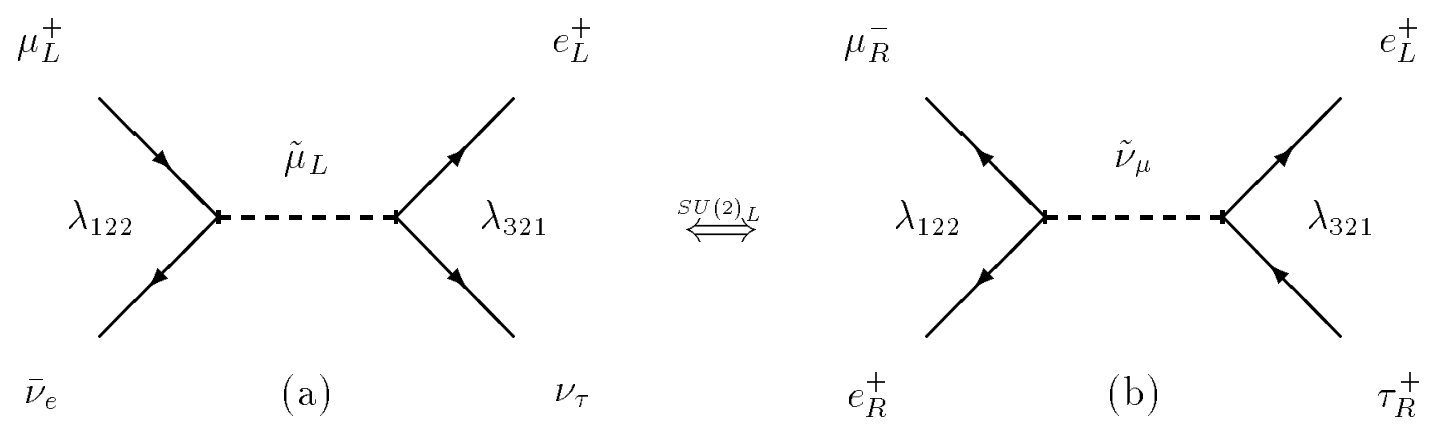

FIG. 6. Diagrams for (a) $\mu^{+} \rightarrow e^{+} \bar{\nu}_{e} \nu_{\tau}$ and (b) $\tau^{-} \rightarrow \mu^{+} e^{-} e^{-}$for SUSY without $R_{p}$ 